\title{
Technical Note: First Use of Endonasal Confocal Laser Endomicroscopy - Feasibility and Proof of Concept
}

\author{
Nina Wenda1 Ralf Kiesslich ${ }^{2}{ }^{10}$ Jan Gosepath ${ }^{10}$ \\ ${ }^{1}$ Department for Otolaryngology, Head and Neck Surgery, Schmidt \\ Kliniken Wiesbaden, Wiesbaden, Germany \\ 2 Department for Gastroenterology, Schmidt Kliniken Wiesbaden, \\ Wiesbaden, Germany
}

Int Arch Otorhinolaryngol 2022;26(3):e396-e400.

\begin{abstract}
Keywords

- confocal laser endomicroscopy

- endonasal endoscopic surgery

- endonasal squamous cell carcinoma

Introduction Probe-based confocal laser endomicroscopy ( $p-C L E)$ is a method for real-time in vivo visualization of mucosal changes on a cellular level. Due to the size of the endoscopes, it was mainly used in the gastrointestinal tract so far. First investigations on head and neck carcinoma described the oropharyngeal application. The further miniaturization of the laser probe now allows endonasal application and, thus, first experiences with the investigation of endonasal neoplasms.

Objectives The aim of the present investigation is to elucidate, based on the morphological criteria validated in the oropharynx, whether these criteria be transferred in a similar way to the endonasal mucosa.

Methods We conducted p-CLE (Cellvizio, Paris, France) with intravenous fluorescein staining in endoscopic sinus surgery in a patient with sinonasal inverted papilloma and a histologically confirmed squamous cell carcinoma. We compared the cellular visualization of pathological changes with those of healthy mucosa in the same specimen, and also with our former findings in the oropharynx.

Results Endonasal p-CLE proved to be quite feasible in the surgical setting, and the transfer of malignancy criteria in analogy to histological examination could be optically retraced. Furthermore, additional criteria for tissue dignity assessment were obtained. Conclusion Our results suggest that endonasal application of $\mathrm{p}-\mathrm{CLE}$ represents a valuable extension of the diagnostic repertoire available to date by an additional realtime analysis of the nasal mucosa. This is of particular value in surgically challenging anatomical areas such as the paranasal sinuses.

Further investigation and validation will be necessary.
\end{abstract}

Address for correspondence Nina Wenda, MD, Ludwig-Erhard-Str. 100, HNO-Ambulanz/ z.H. Fr. Ulrike Berghahn, Wiesbaden, 65199, Germany (e-mail: nina.wenda@helios-gesundheit.de).

\section{Introduction}

Sinonasal malignancies of the head and neck are rare tumors, comprising only $3 \%$ of all head and neck cancers. ${ }^{1}$ Squamous cell carcinoma (SCC) is the most common of these tumors, arising either de novo or in association with inverted papilloma (IP). ${ }^{2}$ received

August 28, 2020

accepted

November 21, 2020

published online

November 5, 2021
DOI https://doi.org/ 10.1055/s-0041-1724091. ISSN 1809-9777.
Depending on the extension of the tumor, the treatment of choice is surgical removal by endoscopic or a combined endoscopic/external approach. ${ }^{3}$ Recurrence, in those arising from IP, most frequently occurs at the initial site of the IP within a period of 2 years after the initial intervention, while late recurrences frequently occur at a different location, being

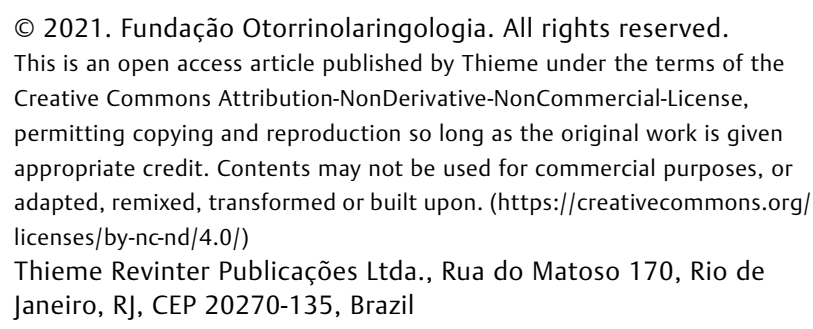

(C) 2021. Fundação Otorrinolaringologia. All rights reserved. This is an open access article published by Thieme under the terms of the Creative Commons Attribution-NonDerivative-NonCommercial-License, permitting copying and reproduction so long as the original work is given appropriate credit. Contents may not be used for commercial purposes, or adapted, remixed, transformed or built upon. (https://creativecommons.org/ licenses/by-nc-nd/4.0/) Thieme Revinter Publicações Ltda., Rua do Matoso 170, Rio de Janeiro, RJ, CEP 20270-135, Brazil 
considered a second tumor. ${ }^{4}$ For most authors, early recurrence implicates incomplete resection. ${ }^{1}$ Clear differentiation of healthy and neoplastic mucosa can rarely be made by endoscopic inspection alone, especially regarding the margins of a lesion.

Real-time information on cellular and subcellular details, especially of the resection margins at the time of the endoscopic examination, could facilitate immediate definitive surgical treatment and, additionally, yield more information on the surrounding tissue.

Confocal laser endomicroscopy (CLE) allows the assessment of changes in the vascular architecture, connective tissue and cellular components of the mucosa in vivo. ${ }^{5}$ This technology was established in the gastrointestinal (GI) tract by researchers of our group using a flexible confocal endomicroscope in order to detect different inflammatory and neoplastic abnormalities in the human colon, esophagus, and stomach. ${ }^{6}$

In 2012, our group described the first oropharyngeal application of this technique and validated malignancy criteria comparing with the gold standard of histological examination. ${ }^{7}$

We present the first endonasal application of in vivo fluorescein-enhanced confocal laser endomicroscopy in IP and SCC to further investigate the use of this technology in the field of functional endoscopic endonasal surgery and tumor resection.

\section{Material and Methods}

The present study was approved by the local ethics committee of Hessen, Germany (No.: FF 146/2017); written informed consent was obtained from each patient before examination.
We performed computer-navigated endoscopic endonasal removal of a right-sided sinunasal IP, as well as of a left sided endonasal squamous cell carcinoma (see - Fig. 1). Probe-based CLE (pCLE) was conducted during these procedures, and fluorescein was used as contrast agent. We followed an algorithm comparing healthy mucosa of the opposite side with the respective lesion in both cases. After detailed documentation, the surgery was completed.

In CLE, a low-power laser is focused to a single point in a microscopic field of view, and the same lens is used as both condenser and objective folding the optical path, so the point of illumination coincides with the point of detection within the specimen. Light emanating from that point is focused through a pinhole to a detector, and light emanating from outside the illuminated spot is rejected from detection.

Illumination and detection systems are at the same focal plane and are termed "confocal". All detected signals from the illuminated spot are captured and measured. The intensity of emitted light is translated into a gray-scale image, which represents microscopic tissue structures. ${ }^{8}$

The evolution of this technology over the past several years has led to the integration of a miniaturized laser scanner into the tip of a conventional flexible video endoscope that can visualize the mucosal details at subcellular resolution during endoscopy.

\section{Results}

Confocal imaging provided instant real-time microscopic imaging during the ongoing surgical procedure. There

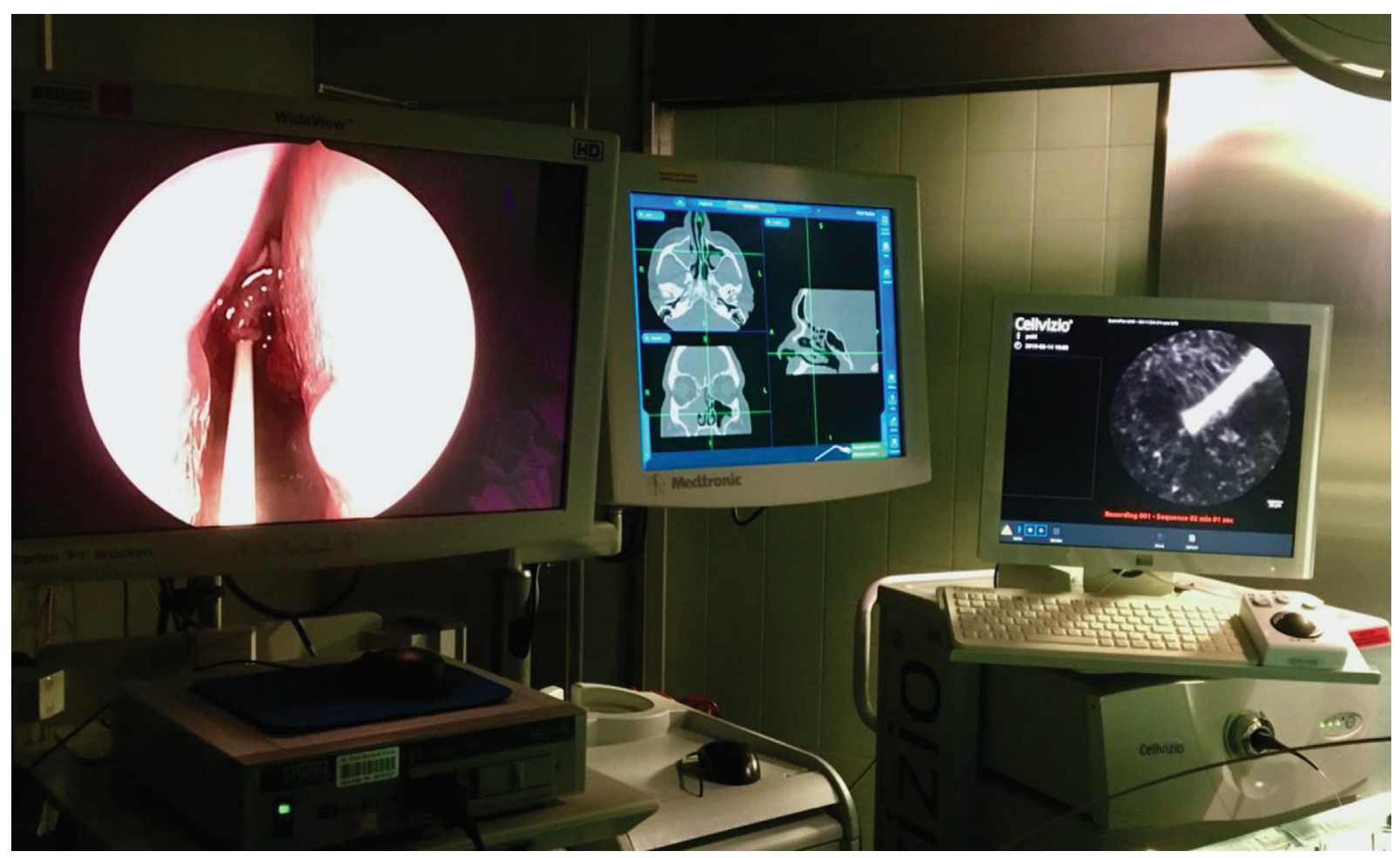

Fig. 1 Intraoperative setting from left to right: AIDA system for functional endoscopic sinus surgery, computed navigation (Medtronic) and confocal laser endomicroscopy (Cellvizio). 

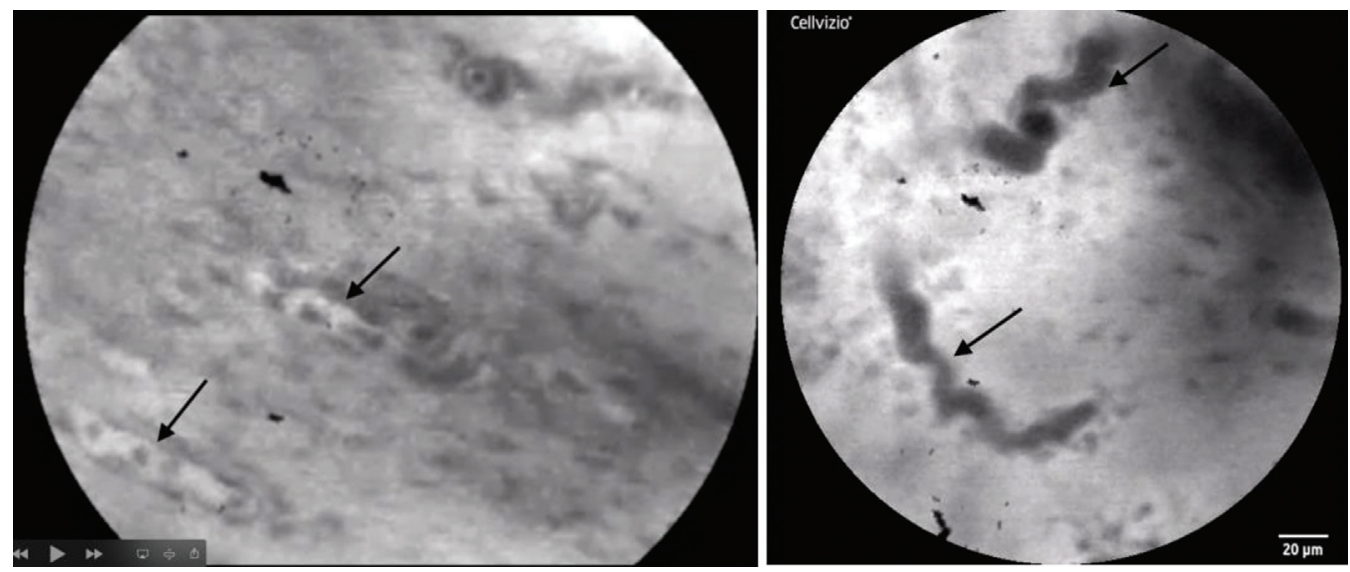

Fig. 2 Left: capillaries with regular and longitudinal configuration and erythrocytes (arrow). Right: extended, "cork-screw-like"-configurated capillaries (arrow).

were no adverse side effects from intravenous fluorescein application after our investigation.

Imaging of the mucosal lesion in the nose as well as in the paranasal sinuses was feasible, and images of good quality could be acquired. Artifacts were most frequently caused by unstable positioning of the probe or by mucus or blood adherent to the probe.

Confocal imaging rarely added $>10$ minutes to the total examination time. Healthy mucosa showed homogenous configuration of the superficial layers, with regular and defined cellular structure. Capillaries displayed regular and longitudinal configuration (see - Fig. 2).

In both entities, the IP and the carcinoma, we visualized an inhomogeneous configuration of the superficial layers with less defined cellular structures and extended, irregular configuration of the capillaries. In squamous cell carcinoma, loss of cellular borders could be seen even more distinctively when compared with IP (see - Fig. 3). - Table 1 displays the morphologic properties for basic screening examination as used in this first report.

Resecting with clear margins was supported by verifying the absence of any of these changes when moving the probe away from the defined lesions. Handling of the probe simultaneously to the endoscope in the narrow conditions of the nose and of the paranasal sinuses was demanding; however, they were quite feasible in the hand of an experienced endoscopicsinus-surgeon. Most areas, even within the maxillary sinus, were accessible, the anterior wall being the most challenging.

\section{Discussion}

This is, to our knowledge, the first report on the intraoperative application of pCLE in neoplasms of the nose and of the paranasal sinuses. Probe-based CLE provides real-time microscopic imaging during ongoing endoscopy and surgical treatment. This advanced technique has been successfully established in many fields, including gastroenterology, urology, dermatology and otolaryngology.., 10

The probe-based system (pCLE, Cellvizio Endomicroscopy System, Mauna Kea

Technologies, Paris, France) consists of a $1.6 \mathrm{~mm}$ flexible miniprobe with a field of view of $240 \mu \mathrm{m}$ diameter, a confocal depth of 55-65 $\mu \mathrm{m}$ and a resolution of $1 \mu \mathrm{m}$.

These probes can be used solo or be fitted through the working channel of most endoscopes for clinical use.

Confocal laser endomicroscopy is only possible using fluorescence contrast agents. Intravenously applied fluorescein sodium $(5 \mathrm{ml}, 10 \%)$ distributes throughout the entire

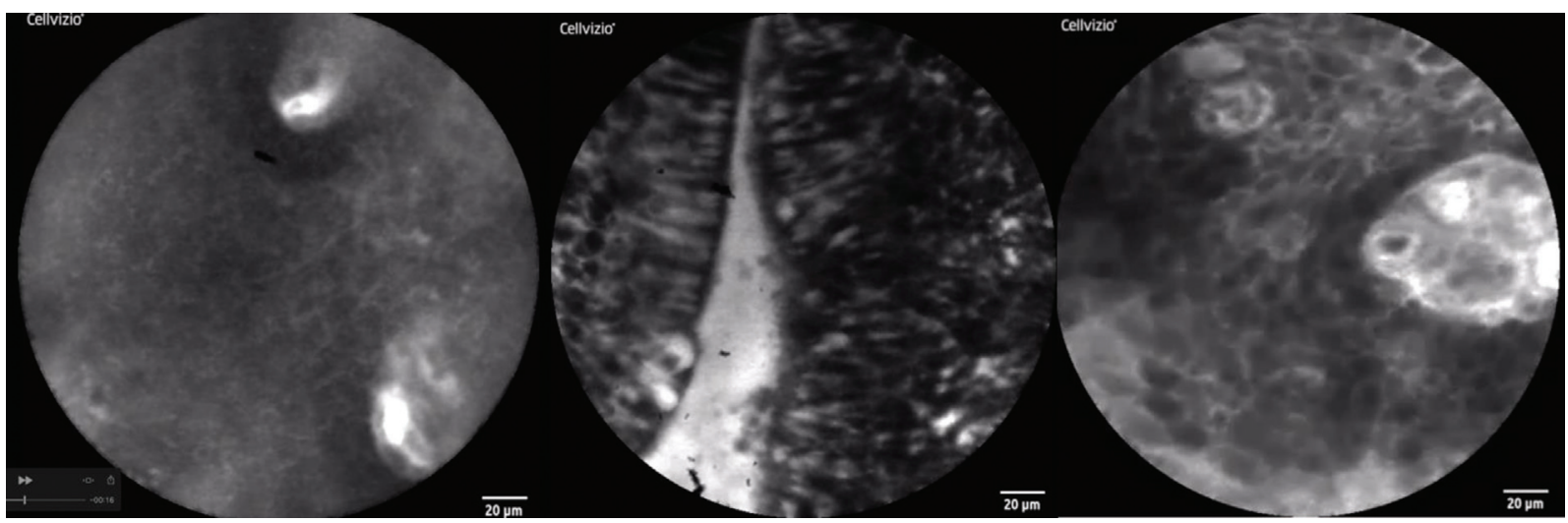

Fig. 3 Left: healthy mucosa: homogenous configuration of the superficial mucosal layer with regular and defined cellular structure and crosssection of two capillaries. Middle: inverted papilloma: superficial epithelial layer with irregular cellular configuration but preserved cell margins. Right: squamous cell carcinoma: inhomogeneous cell structure and extended capillaries. 
Table 1 Morphologic criteria of healthy and neoplastic endonasal mucosa

\begin{tabular}{|l|l|l|}
\hline & healthy mucosa & endonasal neoplasm \\
\hline $\begin{array}{l}\text { Architecture } \\
\text { of tissue }\end{array}$ & $\begin{array}{l}\rightarrow \text { homogeneous configuration } \\
\text { of the superficial layers }\end{array}$ & $\begin{array}{l}\rightarrow \text { nonhomogeneous configuration } \\
\text { of the superficial layers }\end{array}$ \\
\hline $\begin{array}{l}\text { Characteristics } \\
\text { of cells }\end{array}$ & $\begin{array}{l}\rightarrow \text { regular and homogeneous nuclear structure } \\
\rightarrow \text { clearly defined cellular structure }\end{array}$ & $\begin{array}{l}\rightarrow \text { irregular cellular and nuclear structure } \\
\rightarrow \text { blurry cellular morphology }\end{array}$ \\
\hline $\begin{array}{l}\text { Characteristics } \\
\text { of capillaries }\end{array}$ & $\rightarrow$ regular and longitudinal configuration & $\rightarrow$ extended and irregular configuration \\
\hline
\end{tabular}

mucosa with a strong contrast within the connective tissue and the capillary network. Fluorescein binds to serum albumin and the remaining unbound dye molecules pass across systemic capillaries and enter the tissue, highlighting the extracellular matrix. Confocal laser endomicroscopy is possible within seconds after injection. ${ }^{10}$ Intravenous fluorescein is a nontoxic agent that is commonly used in ophthalmology with a long and substantial history of clinical use.

The most common side effects related to the IV administration, which are vomiting and nausea, are transient and minor. More severe side effects, such as vasovagal response, cardiac or respiratory response are extremely rare. ${ }^{11}$

In a pilot study, our group investigated the feasibility of the method using the flexible endoscope in different regions of the human oral cavity and of the oropharynx after intravenous application of fluorescein. ${ }^{12}$ We were able to show for the first time, to our knowledge, that confocal laser endomicroscopy is suitable for the evaluation of epithelial linings of the buccal mucosa, tongue, and floor of the mouth.

Signs of malignancy (altered tissue architecture and increased density of blood vessels with irregular, elongated, and enlarged appearance) could be identified in vivo.

In 2012, our group elaborated a systematic intraoperative evaluation of confocal laser endomicroscopy in the oropharynx. Characteristics of healthy mucosa such as homogenous configuration of the superficial mucosal layer, clearly defined cellular structure, and longitudinal configuration of capillaries could be reproducibly demonstrated in different locations of the head and neck. In SCC, malignancy criteria such as inhomogenous mucosal configuration, blurry cellular borders, as well as extended and irregular configuration of capillaries could be applied. ${ }^{7}$

Based on the current investigation, these criteria appear to be applicable to our endonasal findings.

In 2014, Nathan et al. showed an overall sensitivity for the diagnosis of dysplasia versus nondysplasia of $80.0 \%$ using pCLE in the oral cavity; while the dorsal surface of the tongue was not well visualized, the remaining nonkeratinized subsites, including the buccal mucosa, the floor of mouth and the ventral tongue, were well visualized. ${ }^{13}$

Most recently, Wu et al. showed the application of pCLE in nasopharyngeal carcinoma. ${ }^{14}$ Goncalvez et al. demonstrated the application in vocal cord malignancies, which demonstrated similar characteristics. ${ }^{15}$

The further miniaturization of the probe now allowed nasal passage and examination of the paranasal sinuses.
The application of fluorescein showed to be quite feasible. Tissue was adequately assessable $\sim 30$ seconds after intravenous application. However, the handling of the flexible laser probe is a challenge for the surgeon in the variable conditions of the paranasal sinuses and is subject to an individual learning curve. The necessary mucosal contact pressure of the probe, as well as the contact angle for an adequate visualization, are variable regarding the irregular surfaces of the paranasal sinuses. The guidance of the probe through an instrument such as a straight or angled forceps proved practicable.

The limitations of visualization were particularly seen in increased bleeding, which makes handling and application in well vascularized tumors more demanding.

\section{Conclusion}

The novel application of using p-CLE with miniaturized flexible laser probes appears to be suitable for noninvasive real-time in vivo diagnostics of endonasal lesions. Cellular changes and the extent of resection lines of such lesions can be visualized. Further investigations have to evaluate and validate the significance of this technology in endonasal surgery for invasive malignancy, especially regarding critical resection margins.

Funding

Tumorstiftung Kopf-Hals Wiesbaden, (Grant / Award Number: $15.000 €$ )

Conflict of Interests

The authors have no conflict of interests to declare.

\section{References}

1 Lund VJ, Stammberger H, Nicolai P, et al; European Rhinologic Society Advisory Board on Endoscopic Techniques in the Management of Nose, Paranasal Sinus and Skull Base Tumours. European position paper on endoscopic management of tumours of the nose, paranasal sinuses and skull base. Rhinol Suppl 2010; 22(Suppl.):1-143

2 de Almeida JR, Su SY, Koutourousiou M, et al. Endonasal endoscopic surgery for squamous cell carcinoma of the sinonasal cavities and skull base: Oncologic outcomes based on treatment strategy and tumor etiology. Head Neck 2015;37(08):1163-1169

3 Mirza S, Bradley PJ, Acharya A, Stacey M, Jones NS. Sinonasal inverted papillomas: recurrence, and synchronous and metachronous malignancy. J Laryngol Otol 2007;121(09):857-864

4 Woodworth BA, Bhargave GA, Palmer JN, et al. Clinical outcomes of endoscopic and endoscopic-assisted resection of inverted papillomas: a 15-year experience. Am J Rhinol 2007;21(05):591-600 
400 First Use of Endonasal Confocal Laser Endomicroscopy - Feasibility and Proof of Concept Wenda et al.

5 Kiesslich R, Canto MI. Confocal laser endomicroscopy. Gastrointest Endosc Clin N Am 2009;19(02):261-272

6 Kiesslich R, Burg J, Vieth M, et al. Confocal laser endoscopy for diagnosing intraepithelial neoplasias and colorectal cancer in vivo. Gastroenterology 2004;127(03):706-713

7 Pogorzelski B, Hanenkamp U, Goetz M, Kiesslich R, Gosepath J. Systematic intraoperative application of confocal endomicroscopy for early detection and resection of squamous cell carcinoma of the head and neck: a preliminary report. Arch Otolaryngol Head Neck Surg 2012;138(04):404-411

8 Teubner D, Kiesslich R, Matsumoto T, Rey JW, Hoffman A. Beyond standard image-enhanced endoscopy confocal endomicroscopy. Gastrointest Endosc Clin N Am 2014;24(03):427-434

9 Neumann H, Kiesslich R, Wallace MB, Neurath MF. Confocal laser endomicroscopy: technical advances and clinical applications. Gastroenterology 2010;139(02):388-392, 392.e1-392.e2

10 Wang TD, Friedland S, Sahbaie P, et al. Functional imaging of colonic mucosa with a fibered confocal microscope for real-time in vivo pathology. Clin Gastroenterol Hepatol 2007;5(11): $1300-1305$

11 Jennings BJ, Mathews DE. Adverse reactions during retinal fluorescein angiography. J Am Optom Assoc 1994;65(07): 465-471

12 Haxel BR, Goetz M, Kiesslich R, Gosepath J. Confocal endomicroscopy: a novel application for imaging of oral and oropharyngeal mucosa in human. Eur Arch Otorhinolaryngol 2010;267(03): 443-448

13 Nathan CA, Kaskas NM, Ma X, et al. Confocal Laser Endomicroscopy in the Detection of Head and Neck Precancerous Lesions. Otolaryngol Head Neck Surg 2014;151(01):73-80

$14 \mathrm{Wu}$ L, Yu H,Zhou R, et al. Probe-based confocal laser endomicroscopy for diagnosis of nasopharyngeal carcinoma in vivo. Laryngoscope 2019;129(04):897-902

15 Goncalves M, Aubreville M, Mueller SK, et al. Probe-based confocal laser endomicroscopy in detecting malignant lesions of vocal folds. Acta Otorhinolaryngol Ital 2019;39(06):389-395 